\title{
OPEN Quantitative trait locus mapping identifies the Gpnmb gene as a modifier of mouse macrophage lysosome function
}

\author{
Peggy Robinet ${ }^{1,3,4}$, Brian Ritchey ${ }^{1,4}$, Shuhui Wang Lorkowski ${ }^{1,4}$, Alexander M. Alzayed ${ }^{1}$, \\ Sophia DeGeorgia ${ }^{1}$, Eve Schodowski ${ }^{1}$, C. Alicia Traughber ${ }^{1,2}$ \& Jonathan D. Smith ${ }^{1,2 \bowtie}$
}

We have previously shown that the $\mathrm{DBA} / 2 \mathrm{~J}$ versus $A K R / \mathrm{J}$ mouse strain is associated with decreased autophagy-mediated lysosomal hydrolysis of cholesterol esters. Our objective was to determine differences in lysosome function in AKR/J and DBA/2J macrophages, and identify the responsible genes. Using a novel dual-labeled indicator of lysosome function, DBA/2J versus AKR/J bone marrow derived macrophages had significantly decreased lysosome function. We performed quantitative trait loci mapping of lysosome function in bone marrow macrophages from an AKR/J $\times D B A / 2 \mathrm{~J}$ strain intercross. Four distinct lysosome function loci were identified, which we named macrophage lysosome function modifier (Mlfm) Mlfm1 through Mlfm4. The strongest locus Mlfm1 harbors the Gpnmb gene, which has been shown to recruit autophagy protein light chain 3 to autophagosomes for lysosome fusion. The parental DBA/2J strain has a nonsense variant in Gpnmb. siRNA knockdown of Gpnmb in AKR/J macrophages decreased lysosome function, and Gpnmb deletion through CRISP/Cas9 editing in RAW 264.7 mouse macrophages also demonstrated a similar result. Furthermore, a DBA/2 substrain, called DBA/2J-Gpnmb+/SjJ, contains the wildtype Gpnmb gene, and macrophages from this Gpnmb-preserved DBA/2 substrain exhibited recovered lysosome function. In conclusion, we identified Gpnmb as a causal modifier gene of lysosome function in this strain pair.

Atherosclerosis is a chronic inflammatory disease characterized by the subendothelial accumulation of cholesterol-laden macrophages. Macrophages store excess cholesterol as cholesterol esters (CEs) in lipid droplets, and CE must be hydrolyzed to free cholesterol (FC) to facilitate its efflux, the first step in the reverse cholesterol transport pathway that can promote plaque regression ${ }^{1}$. Although $\mathrm{CE}$ hydrolysis was originally thought to be mediated by cytoplasmic neutral CE hydrolases, it has been demonstrated lysosomal acid lipase plays a significant role in macrophage CE hydrolysis ${ }^{2}$. In this new pathway, lipid droplets are delivered to lysosomes via autophagy, where lysosomal acid lipase hydrolyzes CE to generate FC for cholesterol efflux ${ }^{2}$.

Aortic root atherosclerosis lesion area in apoE-deficient mice is modified by genetic background, with DBA/2J mice having 10-fold larger aortic lesions than AKR/J mice ${ }^{3}$. In cholesterol-loaded bone marrow-derived macrophages (BMDMs) from these two strains, DBA/2J cells accumulate more $\mathrm{CE}$ while $\mathrm{AKR} / \mathrm{J}$ cells have higher FC levels leading to CE/FC ratio 3-fold higher in DBA/2J macrophages ${ }^{4}$. This phenotype appears to be due to effects on both FC esterification ${ }^{5}$, with the AKR strain having an N-terminally truncated Soat 1 gene, encoding the enzyme Acyl-coenzyme A: cholesterol acyltransferase 1 (ACAT1), and on CE hydrolysis ${ }^{4}$. We confirmed the role of autophagy-mediated lysosomal CE hydrolysis in BMDM, and demonstrated that autophagosome fusion with lysosomes is decreased in DBA/2J mice, resulting in impaired autophagic flux and inefficient CE clearance 4 .

In the present study, we applied quantitative trait locus (QTL) mapping to identify genes that impact lysosome function in $\mathrm{BMDM}$ derived from an $\mathrm{AKR} / \mathrm{J} \times \mathrm{DBA} / 2 \mathrm{~J} \mathrm{~F} 4$ strain intercross. We discovered four macrophage lysosome function modifier $(\mathrm{Mlfm})$ loci, with the strongest locus at the proximal region of chromosome $6(\mathrm{Mlfm} 1)$. The Mlfm1QTL harbors the Gpnmb gene, encoding the glycoprotein nonmetastatic melanoma B (GPNMB). Our prior transcriptomic studies of AKR/J and DBA/2J BMDM found Gpnmb mRNA was significantly higher

${ }^{1}$ Department of Cardiovascular and Metabolic Sciences, Lerner Research Institute, Cleveland Clinic, Cleveland, $\mathrm{OH} 44195$, USA. ${ }^{2}$ Department of Molecular Medicine, Cleveland Clinic Lerner College of Medicine of Case Western Reserve University, Cleveland, $\mathrm{OH}$ 44195, USA. ${ }^{3}$ Present address: ProEd Communications, Inc., Beachwood, OH 44122, USA. ${ }^{4}$ These authors contributed equally: Peggy Robinet, Brian Ritchey and Shuhui Wang Lorkowski. ${ }^{\boxplus}$ email: smithj4@ccf.org 

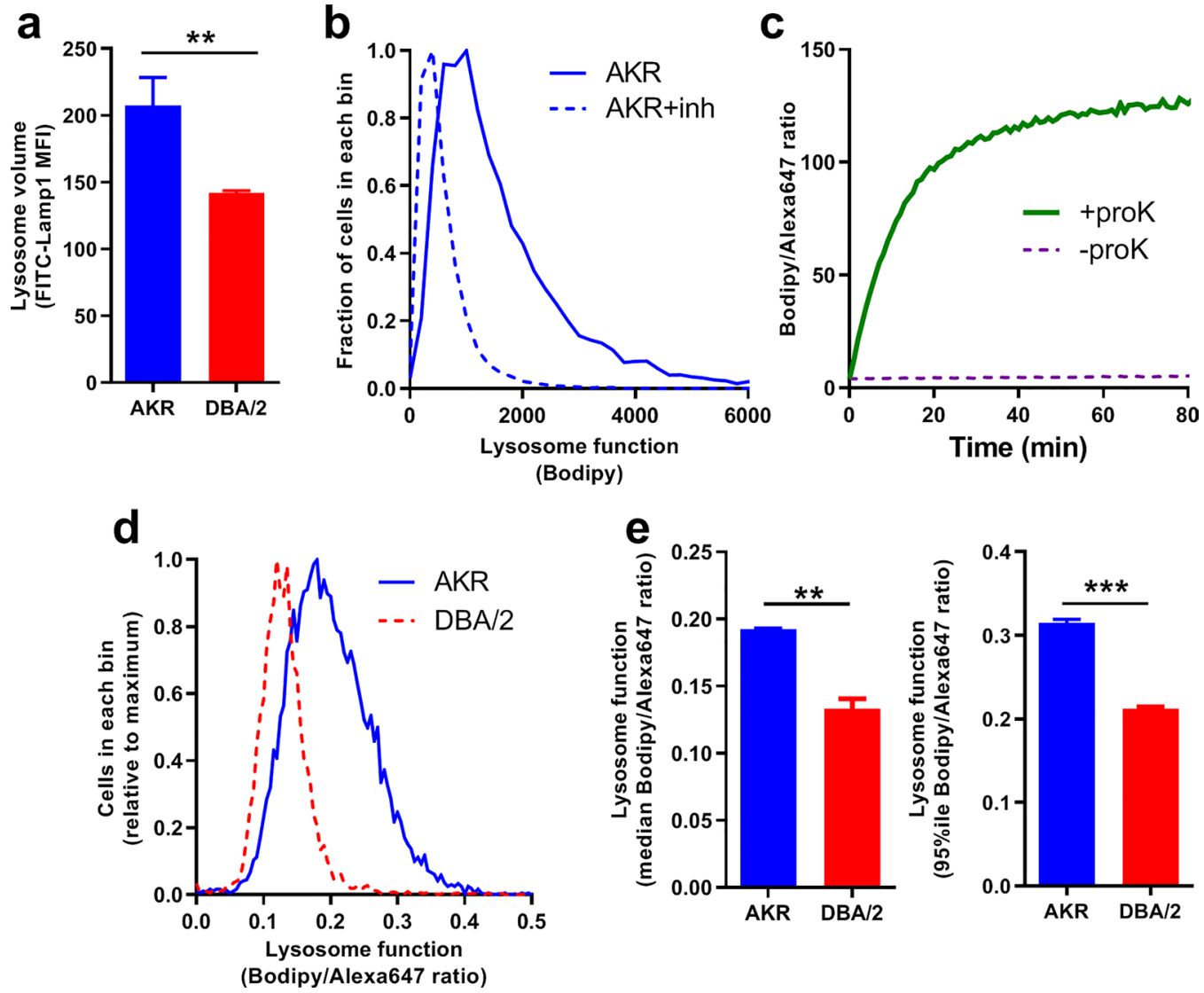

Figure 1. Decreased lysosome function in DBA/2J versus AKR/J macrophages. (a) Lysosome volume was assessed by incubating BMDM with $10 \mu \mathrm{g} / \mathrm{mL}$ FITC-labeled anti-Lampl antibody and examined by flow cytometry (median fluorescence intensity; $\mathrm{n}=3$ per strain, AKR blue bars; DBA/2 red bars). (b) Lysosome function in AKR/J BMDM was assessed by incubating cells with DQ-ovalbumin for $30 \mathrm{~min}$ and examined by flow cytometry (solid line). Lysosome inhibition with $3 \mathrm{~h}$ pretreatment of $10 \mu \mathrm{g} / \mathrm{mL}$ E64d plus $10 \mu \mathrm{g} / \mathrm{mL}$ pepstatin A led a leftward shift (dashed line) indicating decreased lysosome function. (c) Validation of lysosome function indicator in vitro showed proteinase $\mathrm{K}$ increased Bodipy/Alexa647 fluorescence ratio. (d) Lysosome function assay in AKR (solid blue line) and DBA/2 (dashed red line) BMDM was assessed by incubating cells with $2 \mu \mathrm{g} / \mathrm{mL}$ lysosome function indicator for $1 \mathrm{~h}$ and examined by flow cytometry. (e) Analysis of lysosome function in AKR (blue bars) and DBA/2 (red bars) BMDM using median (left panel) or the 95th percentile (right panel) fluorescence intensity ratio (duplicate assay) ${ }^{* *}, p<0.01 ;{ }^{* * *}, p<0.001$ by two-tailed t-test). Graphs prepared using GraphPad Prism software v9.0.0 (www.graphpad.com).

in AKR/J than DBA/2J, and that it's expression was associated with its local genotype resulting in a cis expression QTL (eQTL) ${ }^{6,7}$. It was previously demonstrated that the DBA/2J strain carries a nonsense mutation in the Gpnmb gene ${ }^{8}$. Moreover, GPNMB has been shown to localize on autophagosome and lysosome membranes, and it promotes the recruitment of the autophagy protein light chain 3 (LC3) to the phagosome for lysosomal fusion $^{9}$. Gpnmb expression is also upregulated in lysosomal storage diseases ${ }^{10-12}$, and in macrophages with inflammatory responses ${ }^{13}$. Here, we showed that knockdown of Gpnmb through siRNA in AKR/J macrophages, and Gpnmb deletion by CRISP/Cas9 in RAW 264.7 macrophages, decreased lysosomal function; while, an isocongenic DBA/2 substrain (DBA/2J-Gpnmb +/SjJ) with the wild type Gpnmb gene increased lysosome function compared to DBA/2J BMDM. Therefore, our results validated Gpnmb as the causal lysosomal function modifier gene at the Mlfm1 locus.

\section{Results}

DBA/2J macrophages has decreased lysosome function. DBA/2J versus AKR/J BMDM had less intense lysosomal staining as detected by immunofluoresence using a Lamp1 antibody (Supplemental Fig. 1). This was quantitatively confirmed by flow cytometry where DBA/2J versus AKR/J BMDM showed 32\% decreased Lamp1 staining ( $p<0.01$, Fig. 1a). To investigate whether lysosome function is different in AKR/J and DBA/2J BMDM, we used a commercially available compound, DQ-ovalbumin, in which proteins are heavily labeled with Bodipy leading to fluorescence self-quenching. This compound is taken up by pinocytosis and accumulates in lysosomes, where proteolysis of DQ ovalbumin reduces the quenching, increasing Bodipy fluorescence ${ }^{14}$. We validated that cellular Bodipy fluorescence was blunted by pretreating cells with lysosomal protease inhibitors E64d and pepstatin A (Fig. 1b). However, this assay is also subject to the level of its cellular uptake, thus, we 
a

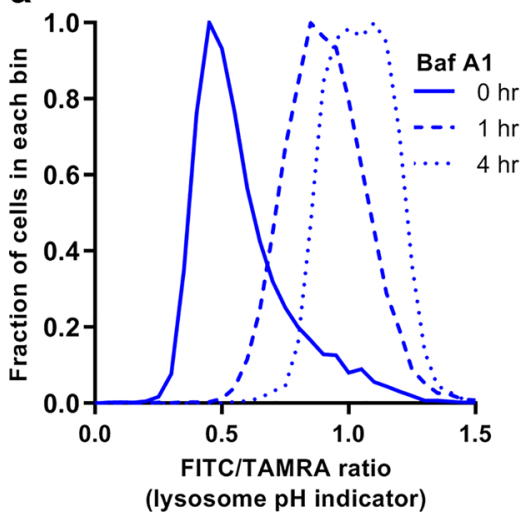

b

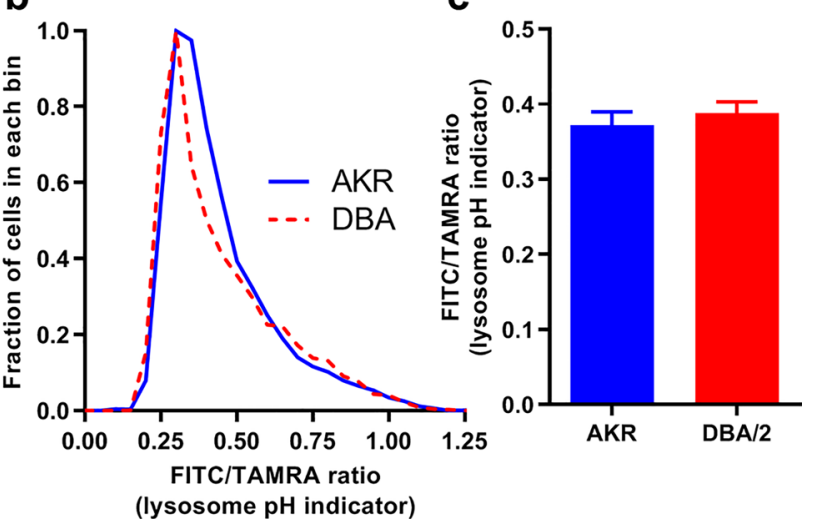

Figure 2. Unaltered lysosomal $\mathrm{pH}$ in DBA/2J versus AKR/J macrophages. (a) AKR BMDMs were incubated with $1 \mathrm{mg} / \mathrm{mL}$ FITC/TAMRA dextran for $18 \mathrm{~h}$, and followed by a $4 \mathrm{~h}$ equilibration $-/+10 \mu \mathrm{g}$ Bafilomycin A1 added for the indicated times. Cells were analyzed by flow cytometry, demonstrating the effectiveness of this probe to assess lysosomal pH. (b) Lysosomal pH assay in AKR (solid blue line) and DBA/2 (dashed red line) BMDM was assessed by incubating cells with FITC/TAMRA dextran and analyzed by flow cytometry. (c) Analysis of lysosomal pH in AKR (blue bars) and DBA/2 (red bars) BMDM using median fluorescence intensity ratio $(\mathrm{n}=3$, not significant by two tailed t-test). Graphs prepared using GraphPad Prism software v9.0.0 (www. graphpad.com).

lightly labeled lysine residues on DQ ovalbumin with Alexa647, allowing us to measure the Bodipy/Alexa647 ratio as an indicator of lysosome function corrected for cellular uptake. This ratiometric lysosome function indicator was validated in vitro, showing that the Bodipy/Alexa647 ratio increased robustly by incubation with proteinase $\mathrm{K}$ (Fig. 1c). We next assessed lysosome function in AKR/J and DBA/2J BMDM by measuring the Bodipy/Alexa647 fluorescence ratio in each cell by flow cytometry and plotting the frequency distribution of this ratio in $\approx 10,000$ cells (Fig. $1 \mathrm{~d}$ ). The median Bodipy/Alexa647 ratio was $45 \%$ higher in in AKR/J versus DBA/2J $(p<0.01$, Fig. 1e left panel), while the 95th percentile was $49 \%$ higher in AKR/J versus DBA/2J $(p<0.001$, Fig. 1e right panel); demonstrating decreased lysosome function in DBA/2J BMDM.

We then measured the lysosomal $\mathrm{pH}$ using commercially available FITC/TAMRA dual-labeled dextran, which is taken up by pinocytosis and accumulates in lysosomes. FITC fluorescence is $\mathrm{pH}$-sensitive and is decreased as $\mathrm{pH}$ reduces in acidic organelles such as lysosomes, while TAMRA fluorescence is $\mathrm{pH}$-insensitive. Thus, cellular FITC/TAMRA ratio is an indicator of lysosomal $\mathrm{pH}$. We demonstrated the specificity of this assay by treatment with bafilomycin A1, a highly specific V-ATPase inhibitor, which led to increased FITC/TAMRA ratio in a time-dependent manner (Fig. 2a). However, there was no difference in FITC/TAMRA ratio comparing AKR/J and DBA/2J BMDM, indicating that lysosomal $\mathrm{pH}$ was not statistically different in those two strains (Fig. 2b,c). Therefore, we showed that DBA/2J versus AKR macrophages have decreased lysosome function, which cannot be attributed to increased lysosomal $\mathrm{pH}$.

Significant QTL for lysosome function maps to chromosome 6. We used a genetic approach to identify the gene region responsible for the strain difference in lysosome function. Lysosome function was measured in BMDM from $120 \mathrm{AKR} / \mathrm{J} \times \mathrm{DBA} / 2 \mathrm{~J} F 4$ mice by flow cytometry after incubation with the Bodipy/Alexa647 ovalbumin indicator; and, the Bodipy/Alexa647 ratio histograms were plotted for each BMDM from each F4 mouse. For genetic analysis we used the Bodipy/Alexa647 ratio 95th percentile values, as these were more significant than the median values (Fig. 1e). There was no significant effect of sex on this phenotype $(p=0.61)$, so we combined data from male and female BMDM. The lysosome function values for the F4 mice were normally distributed (Fig. 3a) and were used to perform QTL mapping. Two distinct macrophage lysosome function QTLs were identified on the proximal regions of chromosomes 6 and 17, and we named them macrophage lysosome function modifier (Mlfm) Mlfm 1 and $M l f m 2$ (Fig. 3b). A similar LOD plot reproducing Mlfm 1 and Mlfm2, along with a new peak on the X chromosome, was obtained using the median Bodipy/Alexa647 ratio (Supplemental Fig. 2); however, the LOD peaks were not as significant, showing that the 95th percentile values, which may represent maximal lysosomal function from each F4 BMDM, yielded a better quantitative trait. The stronger Mlfml QTL was located at $49.7 \mathrm{Mb}$ on chromosome 6 (90\% confidence interval 28.7-64.9 Mb), which had the logarithm of the odds (LOD) score of 6.09 (Table 1). There was a gene dosage effect of Mlfm 1 on lysosome function with each DBA/2J allele decreasing lysosome function by 6\% (Fig. 3c, ANOVA linear trend test $\mathrm{r}^{2}=0.208, p<0.0001$ ), indicating that this locus is associated with $\sim 21 \%$ of the variance in lysosome function in the F4 cohort. Mlfm2 was located at $9.5 \mathrm{Mb}$ on chromosome 17 (90\% confidence interval $6.0-16.5 \mathrm{Mb}$ ) with a LOD score of 4.28 (Table 1). After adjusting for the $M l f m 1$ genotype as an additive covariate, new Mlfm3 and Mlfm4 loci were identified on the distal sides of chromosomes 2 and 17 (Fig. 4), with Mlfm3 and Mlfm4 LOD scores of 5.55 and 4.32, respectively (Table 2). Mlfm2 on the proximal end of chromosome 17 was moderately strengthened after this adjustment (LOD score 4.61, Table 2). 
a

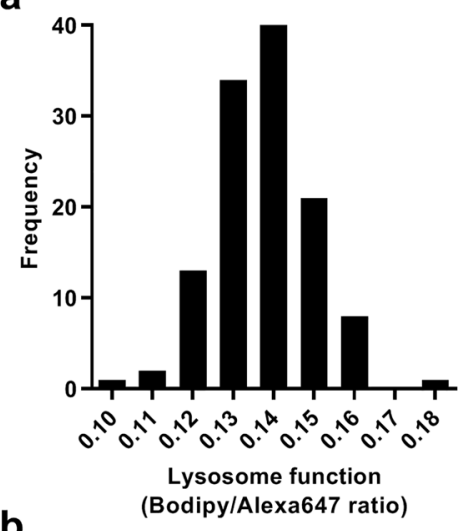

(Bodipy/Alexa647 ratio)
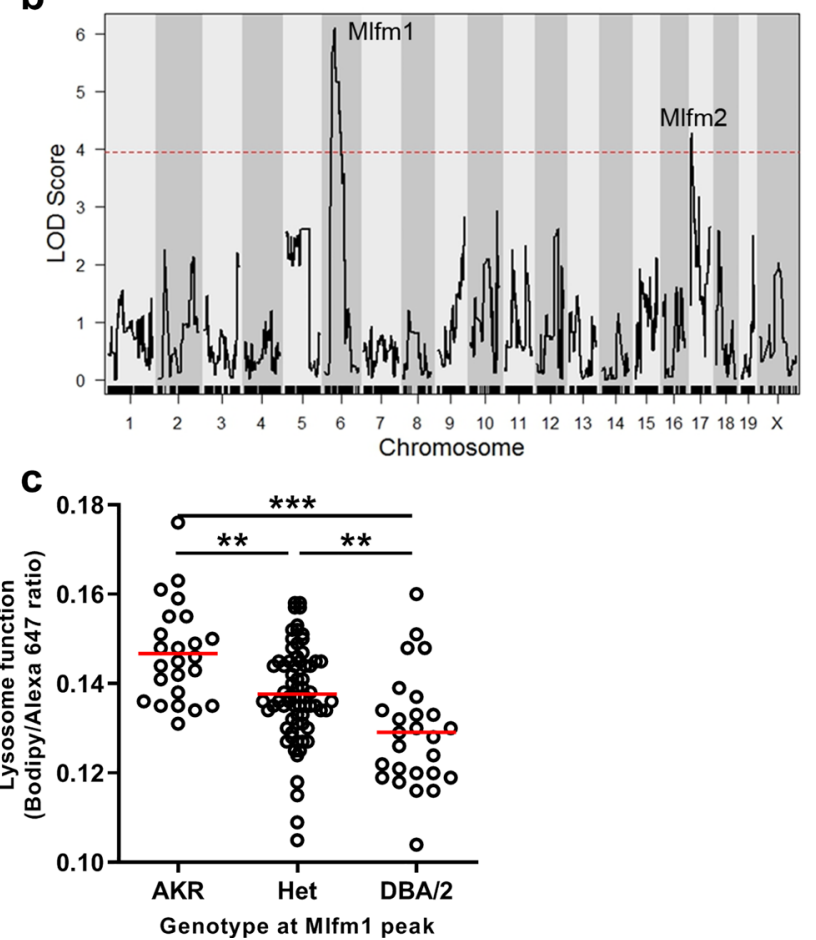

Figure 3. QTL analysis for lysosome function. (a) Normal distribution of the Bodipy/Alexa647 fluorescence ratio after F4 BMDM incubation with lysosome function indicator. (b) QTL LOD plot for lysosome function showing Mlfm 1 and Mlfm2 on chromosomes 6 and 17, respectively. The dashed red line shows the genome-wide $p=0.05$ threshold based on 10,000 permutations. (c) F4 BMDM lysosome function values by genotype at the $M f l m 1$ peak marker (mean values [red lines]; ANOVA linear trend test $r^{2}=0.208, p<0.0001 ;{ }^{* *}, p<0.01$, and ${ }^{* * *}$, $p<0.001$ by ANOVA Tukey posttest). QTL plot prepared using r/QTL ${ }^{44}$, other graphs prepared using GraphPad Prism software v9.0.0 (www.graphpad.com).

\begin{tabular}{|l|l|l|l|l|}
\hline QTL name & Chromosome & Peak Mb (90\% confidence interval) & Max LOD & Genome wide $\boldsymbol{p}$ value \\
\hline lfm 1 & 6 & $49.7(28.7-64.9)$ & 6.09 & $<0.05$ \\
\hline Mlfm2 & 17 & $9.5(6.0-16.5)$ & 4.28 & $<0.05$ \\
\hline
\end{tabular}

Table 1. Macrophage lysosome function modifier QTLs.

Identification of Gpnmb as the gene responsible for the Mlfm1 QTL. We next performed a Bayesian analysis for lysosome function at Mlfm1 yielding a $90 \%$ confidence interval of 25.89 to $63.78 \mathrm{Mb}$, which contained 430 genes. Among them, Gpnmb, mapping at $48.99 \mathrm{Mb}$ (0.17 Mb from the LOD peak), has a C>T mutation in DBA/2J mice leading to an early stop codon in exon $4^{8}$. This mutation is predicted to lead to non-sense mediated mRNA decay as traditionally defined ${ }^{15}$. We previously observed a DBA/2J-AKR/J strain difference in BMDM Gpnmb mRNA levels in a microarray study with DBA/2J macrophages expressing 12.5 fold less Gpnmb 


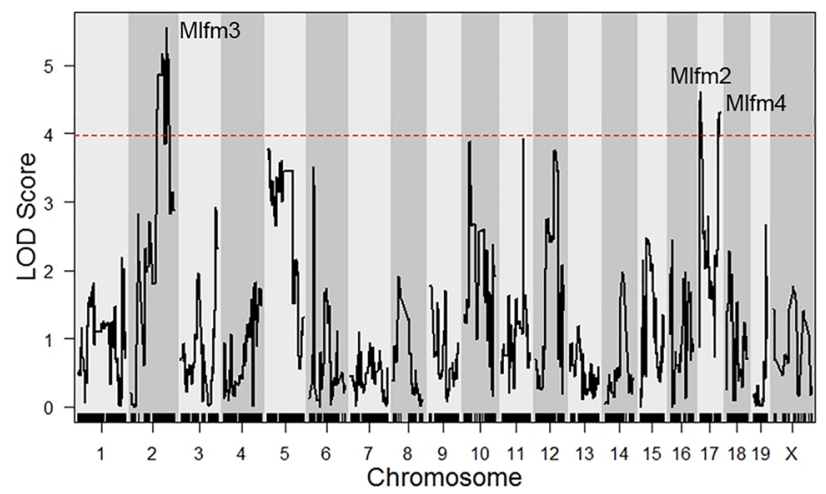

Figure 4. QTL mapping for lysosome function after adjusting for Mlfml as an additive co-variate. The dashed red line shows the genome-wide $p=0.05$ threshold based on 10,000 permutations. QTL plot prepared using $\mathrm{r} /$ $\mathrm{QTL}^{44}$.

\begin{tabular}{|l|l|l|l|l|}
\hline QTL name & Chromosome & Peak Mb $(\mathbf{9 0} \%$ confidence interval) & Max LOD & Genome wide $\boldsymbol{p}$ value \\
\hline Mlfm 2 & 17 & $9.6(6.0-17.1)$ & 4.61 & $<0.05$ \\
\hline Mlfm3 & 2 & $147.5(111.2-158.1)$ & 5.55 & $<0.05$ \\
\hline Mlfm4 & 17 & $87.5(79.4-89.1)$ & 4.32 & $<0.05$ \\
\hline
\end{tabular}

Table 2. Macrophage lysosome function modifier QTLs after adjusting for Mlfm1.

$\mathrm{mRNA}^{6}$; and, our prior independent $\mathrm{AKR} / \mathrm{J} \times \mathrm{DBA} / 2 \mathrm{~J}$ F2 strain intercross demonstrates a strong cis eQTL for Gpnmb expression in BMDM with a LOD score of $22^{7}$. Gpnmb codes for the GPNMB protein, which has been shown to be responsible for the recruitment of LC3 to the phagosome for lysosomal fusion?.

With Gpnmb being the strongest Mlfm1 candidate modifier gene for lysosome function, we first performed Western blot in AKR/J and DBA/2J BMDM to confirm that there was no GPNMB expression detectable in DBA/2J macrophages (Fig. 5a). To determine whether the Gpnmb is responsible for the Mlfm1 QTL for lysosome function, we used a DBA/2 coisogenic substrain, called DBA/2J-Gpnmb $/$ SjJ (referred to as DBA/ $2 \mathrm{~g}^{+}$), which was separated from the main DBA/2J line before the Gpnmb ${ }^{R 150 X}$ null allele arose, and is backcrossed to the modern DBA/2J to maintain the wildtype Gpnmb allele on the DBA/2J background ${ }^{16}$. We confirmed GPNMB protein expression in this line by Western blot (Fig. 5a left side). Furthermore, we used siRNA to knockdown Gpnmb in $\mathrm{AKR} / \mathrm{J}$ BMDM (referred to as $\mathrm{AKRg}^{-}$), with GPNMB protein expression decreased by $79 \%$ (Fig. 5a right side). We next measured lysosome function in AKR/J, AKRg-, DBA/2J and DBA/2 $\mathrm{g}^{+} \mathrm{BMDM}$ using the Bodipy/ Alexa647 ovalbumin lysosome function indicator (Fig. 5b). We confirmed DBA/2J versus AKR/J BMDM had a $27 \%$ decrease in lysosome function $(p<0.001$, by ANNOVA posttest). AKRg- versus AKR/J BMDM had a $12 \%$ decrease in lysosome function $(p<0.001)$, and $\mathrm{DBA} / 2 \mathrm{~g}^{+} \mathrm{BMDM}$ restored lysosome function to the comparable level of AKR/J BMDM (DBA2/g+ vs. DBA/2J, 30\% increase, $p<0.001 ; \mathrm{DBA} / 2 \mathrm{~g}^{+}$vs. AKR/J, not significant). These findings indicate that the DBA/2J Gpnmb nonsense mutation allele is responsible for the Mlfm1 QTL, and thus plays a major role in the AKR/J-DBA/2J strain effect on lysosome function. To replicate this effect in a different genetic background, we used CRISPR/Cas9 in the RAW264.7 macrophage cell line (referred to as RAW), derived from the BALB/c strain, to delete the entire Gpnmb gene (referred to as RAWg-). We confirmed lack of GPNMB protein expression in RAWg- by Western blot (Fig. 6a). RAWg- versus RAW macrophages had 19\% decreased lysosome function $(p<0.0001$, Fig. 6b). However, in the presence of LPS pretreatment, RAWg-versus RAW macrophages had $25 \%$ decreased lysosome function $(p<0.0001$, Fig. $6 \mathrm{~b}$ ). These data confirm that Gpnmb expression increased lysosome function and supports our finding that Gpnmb was responsible for the Mlfm1 QTL.

\section{Discussion}

We previously crossed apoE-deficiency onto six inbred strains, DBA/2J, C57BL/6J, 129/SV-ter, AKR/J, BALB/ $\mathrm{cByJ}$, and $\mathrm{C} 3 \mathrm{H} / \mathrm{HeJ}$; and, among these strains the $\mathrm{DBA} / 2 \mathrm{~J}$ has the largest aortic root atherosclerotic lesions, while AKR/J was one of several strains with small lesions ${ }^{3}$. This led us to follow up with two independent strain intercrosses to identify atherosclerosis modifier genes using the DBA/2J and AKR/J parental strains, which identified three confirmed atherosclerosis QTLs, Ath22, Ath26, and Ath $28^{7,17}$. Since macrophages are a key cell type in atherogenesis, we also performed eQTL analysis of BMDM from these same two independent $\mathrm{AKR} / \mathrm{J} \times \mathrm{DBA} / 2 \mathrm{~J}$ strains intercrosses in order to gain insights into potential atherosclerosis modifier candidate genes $^{7,18}$. We also started series of studies to explore BMDM phenotypes from these two parental strains. Thus far we found significant strain effects on cholesterol ester loading, cholesterol efflux to apolipoprotein A1 or HDL acceptors, autolysosome formation ${ }^{4}$, and, in the present study, lysosome function. To identify QTL loci for these traits, we bred an F4 strain intercross and froze down aliquots of bone marrow for subsequent phenotype 
a

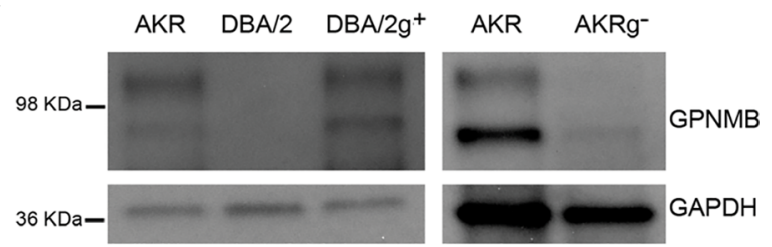

b

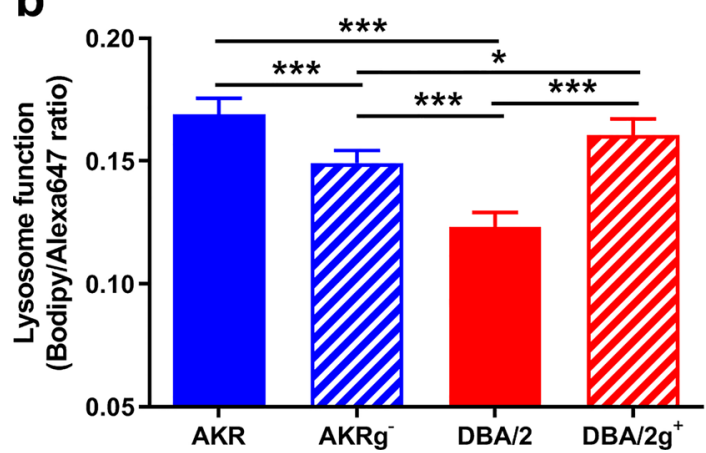

Figure 5. Altered lysosome function dependent upon Gpnmb expression. (a) GPNMB and GAPDH western blot from lysates of AKR, DBA/2, and DBA/2g + BMDM (left panel); and from AKR BMDM transfected with control or Gpnmb siRNA (right panel). Uncropped blots shown in Supplemental Figs. 3, 4. (b) Lysosome function was assessed by $1 \mathrm{~h}$ incubation of $2 \mu \mathrm{g} / \mathrm{mL}$ lysosome function indicator with BMDM from AKR control siRNA (solid blue bar), AKRg (striped blue bar), DBA/2 (solid red bar), and DBA $/ 2 \mathrm{~g}^{+}$(striped red bar). Results were illustrated using median fluorescence intensity ratio $\left(\mathrm{n}=5 ;{ }^{*}, p<0.05 ;{ }^{* *}, p<0.001\right.$ by ANOVA with Tukey posttest). Graph prepared using GraphPad Prism software v9.0.0 (www.graphpad.com).
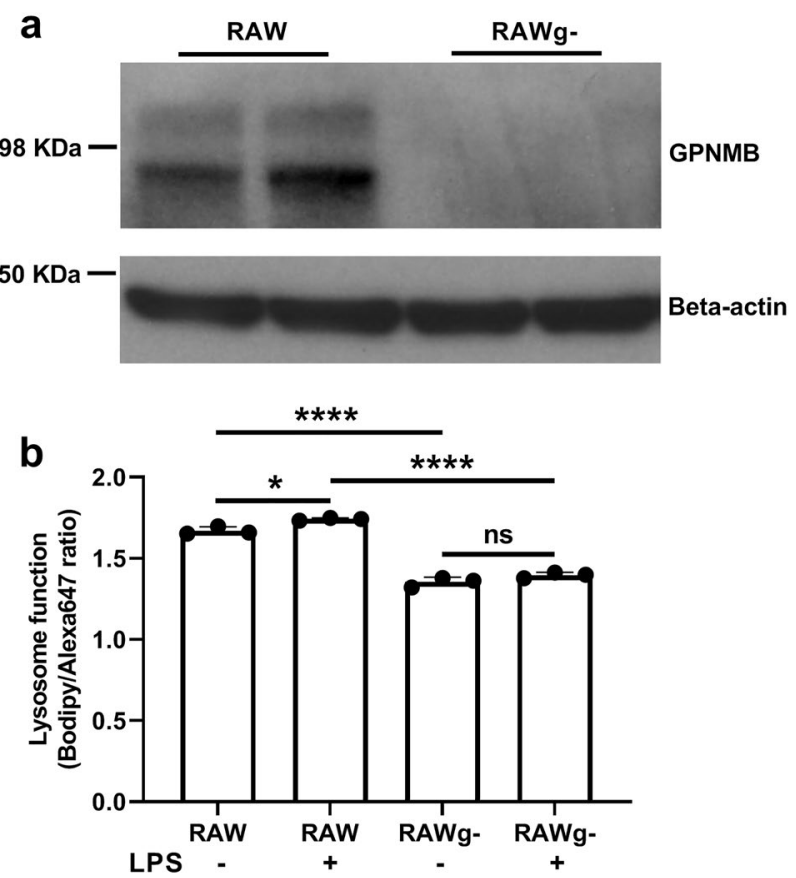

Figure 6. Decreased lysosome function in Gpnmb knockout RAW macrophages. (a) GPNMB western blot from lysates of RAW264.7 macrophages and Gpnmb knockout RAW264.7 macrophages. Uncropped blot shown in Supplemental Fig. 5. (b) Lysosome function was assessed by $1 \mathrm{~h}$ incubation of $2 \mu \mathrm{g} / \mathrm{mL}$ lysosome function indicator with RAW264.7 and Gpnmb knockout RAW264.7 macrophages with or without $24 \mathrm{~h}$ pretreatment with $250 \mathrm{ng} / \mathrm{mL}$ LPS. Results were illustrated using median fluorescence intensity ratio $\left({ }^{*}, p<0.05\right.$; ${ }^{* * * *}$, $p<0.0001$ by ANOVA with Tukey's posttest). Graph prepared using GraphPad Prism software v9.0.0 (www. graphpad.com). 
studies. We recently reported the first of these QTL studies, which identified an AKR/J deletion in exon 2 of the Soat1 gene, encoding acyl-CoA:cholesterol acyl transferase 1, also known as ACAT1, as the strongest locus modifying cholesterol ester loading ${ }^{5}$.

The DBA/2J and AKR/J inbred strains have been useful in many areas of mouse physiology and disease. For example, the DBA/2J strain is susceptible to epicardiac calcification, which was mapped to the Dyscalc1 QTL; and, the causal gene was identified $A b c c 6$ gene which has undetectable expression in the DBA/2J strain ${ }^{19}$. Another feature of the DBA/2J strain is that $~ 70 \%$ of these mice develop glaucoma by 12 months of age, after iris pigment dispersion (ipd) and iris stromal atrophy (isa) ${ }^{16}$. In a DBA/2J $\times$ C57BL/6J strain intercross, the ipd and isa phenotypes segregated to the ipd locus on chromosome 6 and the isa locus on chromosome $4^{20}$. Subsequent $\mathrm{DBA} / 2 \mathrm{~J} \times \mathrm{CAST} /$ Ei intercrosses fine mapped the ipd locus, and led to the discovery that this phenotype was due to a nonsense mutation in the Gpnmb gene $\left(G p n m b^{R 150 X}\right)$ in the DBA/2J strain ${ }^{8}$. Furthermore, DBA/2 substrains with the wildtype Gpnmb gene do not have the ipd phenotype ${ }^{8}$. Thus, the same DBA/2J Gpnmb nonsense allele responsible for decreased macrophage lysosome function in our study is responsible for the ipd phenotype in the eye.

Gpnmb encodes for Glycoprotein Non-Metastatic Protein B (GPNMB) which was originally discovered in a melanoma cell line ${ }^{21}$. This protein, also called osteoactivin, DC-HIL, or hematopoietic growth factor inducible neurokinin-1, has been studied extensively in many contexts including cancer, kidney injury, obesity, nonalcoholic steatohepatitis, Parkinson disease, osteoarthritis, lysosome storage disorders, and heart failure; and, in most of these contexts expression of GPNMB is induced by the related pathology, likely in response to lysosomal stress $^{10,22-27}$. However, loss of Gpnmb expression in DBA/2J mice is associated with preserved cardiac function after myocardial infarction ${ }^{28}$. The role of GPNMB/osteoactivin in bone formation, osteoblasts, and osteoclasts has been studied extensively. DBA/2 versus C57BL/6 mice have smaller femoral cross section area and study of an F2 cohort from intercrossing these two strains identified four gender-independent QTLs for this trait, including one on chromosome 6 where the Gpnmb gene resides ${ }^{29}$. Various transgenic models of GPNMB/osteoactivin over expression have been studied for effects on bone morphology and osteoclast number and function, with some discrepancy between non-tissue specific expression and osteoclast specific expression ${ }^{30-32}$. More relevant to the current study, DBA/2 and DBA/2g + mice have been assessed for bone phenotypes. DBA/ 2 versus DBA2 $g+$ mice have decreased femur bone cross sectional area/mass, marrow area, and cortical bone porosity associated with decreased osteoblast differentiation, proliferation, and survival, as well as with decreased osteoclast function, but increased osteoclast differentiation ex vivo ${ }^{33,34}$. Thus, there is much interest in Gpnmb and the role it plays in a multitude of diseases and in normal physiology.

In the current study, we verified that the Gpnmb null allele in the DBA/2J strain was likely responsible for the Mlfm1 QTL on chromosome 6, the strongest locus for macrophage lysosome function. In addition to the Mlfm1 QTL, we identified 3 other Mlfm loci on the distal region of chromosome $2(\mathrm{Mlfm} 3)$ and on the proximal and distal regions of chromosome 17 (Mlfm2 and Mlfm4). Further study will be needed to elucidate whether Gpnmb, or the genes at the other Mlfm QTLs, can account for the decreased autolysosome formation and lipid droplet turnover observed in DBA/2J macrophages.

We were able to identify the likely causal gene for the Mlfm1 QTL without laborious breeding of congenic strains for fine mapping. However, it is possible that other genes affecting lysosome function may also map to Mlfm1 QTL; but, the most parsimonious explanation is that the Gpnmb gene is responsible for this QTL. Our success in this endeavor was aided by several factors including the use of an F4 intercross cohort leading to more recombinations per chromosome and by performing a high density genome scan leading to a precise QTL peak. Another fortuitous factor was the availability of the DBA/2 iso-congenic substrain expressing wildtype Gpnmb. In our experience several phenotype assays performed in mice have high coefficients of variation. For example, fatty streak aortic root lesion areas in 16 week old chow diet-fed apoE-deficient mice on inbred background strains often yield coefficients of variation approaching 50\%. This large phenotypic variation, due to either stochastic or subtle environmental differences, leads to decreased power to detect QTLs. In the current study, we used ex-vivo cell based assays, which had smaller coefficients of variation of $\sim 10 \%$, leading to better power for QTL mapping even with a smaller sample size compared to many mouse QTL studies. We call this ex vivo cell based approach 'QTL in a dish'. One advantage of this method is the ability to treat cells with compounds or conditions that would be difficult to perform or painful in live mice. This 'GWAS in a dish' method is also being used to study phenotypes in different cell types derived from the differentiation of human induced pluripotent stem cells originating from cohorts consisting of $\sim 100$ to 200 subjects $^{35-37}$.

\section{Methods}

Mouse strains. AKR/J, DBA/2J, and DBA/2J-Gpnmb ${ }^{+} / \mathrm{SjJ}$ (stock \# 007048) mice were obtained from JAX. The DBA/2J-Gpnmb ${ }^{+} / \mathrm{SjJ}$ coisogenic mice were from the DBA/2 Sandy substrain, which was separated from the main DBA/2J line in the early 1980s, before the Gpnmb ${ }^{R 150 X}$ null allele was fixed in the DBA/2J stock. Modern backcrossing to DBA/2J mice was performed to maintain the wildtype $G p n m b\left(\mathrm{~g}^{+}\right)$allele on the DBA/2J background $^{16}$. All mouse studies were approved by the Cleveland Clinic Animal Care and Use Committee, and all mouse studies were performed in accordance with the approved protocol. Mouse studies were carried out in compliance with the ARRIVE guidelines (http://www.nc3rs.org.uk/page.asp?id=1357).

Generation and genotyping of AKR/J $\times D B A / 2 J$ F4 mice. Parental male AKR/J and female DBA/2J mice were crossed to generate the F1 generation, fixing the $\mathrm{Y}$ chromosome from the AKR/J strain. Two breeding pairs of F1 mice were bred to generate the F2 mice, and two breeding pairs of F2 mice were used to generate F3 mice. Six breeding pairs of F3 mice were used to generate the $122 \mathrm{~F} 4$ mice, which consisted of 70 males and 52 females ${ }^{5}$. Healthy F4 mice were sacrificed at 8-10 weeks of age. Ear tissue was collected from each mouse and 


\begin{tabular}{|l|l|l|}
\hline sgRNA & Sequence & Position on Chr. 6 \\
\hline musgGpnmb\#1 & ACCAACGACCAGGUUUCGUU & $49,036,355-49,036,374$ \\
\hline musgGpnmb\#2 & GUGCATCGCCUUCAAACUAU & $49,058,250-49,058,269$ \\
\hline
\end{tabular}

Table 3. sgRNAs used to delete the mouse Gpnmb gene.

digested overnight at $55^{\circ} \mathrm{C}$ in lysis buffer containing $20 \mathrm{mg} / \mathrm{mL}$ proteinase $\mathrm{K}$. DNA was ethanol precipitated and resuspended in $10 \mathrm{mM}$ Tris $1 \mathrm{mM}$ EDTA $(\mathrm{pH}=8)$. Femurs were promptly flushed after sacrifice, and bone marrow cells were washed, aliquoted, and cryopreserved. Cells were thawed and differentiated into macrophages at the time of experimentation, as described below. F4 mice were genotyped as described previously ${ }^{5}$. Briefly, the GeneSeek MegaMUGA SNP array was used, and filtering for call frequency and strain polymorphism using parental and $F_{1}$ DNA yielded 16,975 informative SNPs that were used for QTL mapping. All marker locations are based on NCBI Mouse Genome Build 37.

Bone marrow-derived macrophages. Bone marrow derived macrophages were obtained from female and male F4 mice (two were excluded due to low yields) and female mice on the AKR/J, DBA/2J and DBA/2 ${ }^{+}$ background. Bone marrow cells were suspended in macrophage growth medium (DMEM, 10\% FBS, 20\% L-cells conditioned media as a source of MSCF) as previously described ${ }^{38,39}$ and plated in tissue culture coated 6, 12, or 24 well plates. The media was renewed twice per week. Cells were used for experiments 10 to 14 days after plating when the bone marrow cells were confluent and fully differentiated into macrophages. When required, AKR/J cells were transfected with $50 \mathrm{nM}$ silencer-select Gpnmb (4390771, Thermofisher Scientific) or control (4390843, Thermofisher Scientific) siRNA using TransIT-TKO (MIR2150, Mirus) as described by the manufacturer. Cells were incubated with the siRNA complexes for $48 \mathrm{~h}$, media was then replaced with fresh macrophage growth media (in the presence or absence of $50 \mu \mathrm{g} / \mathrm{mL}$ AcLDL as indicated), and incubated for another $24 \mathrm{~h}$ before experiments.

Lipoprotein preparations. Human LDL $(1.019<\mathrm{d}<1.063 \mathrm{~g} / \mathrm{mL})$ were prepared by ultracentrifugation from de-identified expired blood bank human plasma (reviewed by the Cleveland Clinic Institutional Review Board and found exempt from human research rules). LDL was acetylated as described previously ${ }^{40,41}$ and dialyzed against PBS with $100 \mu \mathrm{M}$ EDTA and $20 \mu \mathrm{M}$ BHT. Protein concentrations of lipoproteins were determined using an alkaline Lowry assay ${ }^{42}$. When indicated, cells were loaded with $50 \mu \mathrm{g} / \mathrm{mL}$ of AcLDL for $24 \mathrm{~h}$.

Lysosome volume assay. Macrophages were first stained and gated for live cells with LIVE/DEAD Fixable Blue Dead Cell Stain (L23105, Thermofisher Scientific). The cells were fixed in $4 \%$ paraformaldehyde and permeabilized with saponin, lysosomes were labeled using $10 \mu \mathrm{g} / \mathrm{mL}$ dilution of FITC-labeled antibody against mouse Lamp-1 (ab24871, abcam), a lysosomal structural protein, and detected by flow cytometry.

Lysosome function assay. To validate the use of DQ-ovalbumin as a surrogate measure of lysosome function, cells were pre-treated for $3 \mathrm{~h}$ in absence or presence of $10 \mu \mathrm{g} / \mathrm{mL}$ E64d (E8640, Sigma-Aldrich) and 10 $\mu \mathrm{g} / \mathrm{mL}$ pepstatin A (P5318, Sigma-Aldrich) before incubating for $30 \mathrm{~min}$ with the reagent. To make lysosome function indicator, $1 \mathrm{mg}$ of DQ-ovalbumin (D12053, Thermofisher Scientific) in $0.1 \mathrm{M}$ sodium bicarbonate was incubated with $98 \mu \mathrm{g}$ of Alexa Fluor 647 succinimidyl ester (A20006, Thermofisher Scientific) for $1 \mathrm{~h}$ at room temperature (3:1 dye:protein mole ratio). The reaction was stopped by incubating the conjugate with $0.1 \mathrm{~mL}$ of $1.5 \mathrm{M}$ hydroxylamine ( $\mathrm{pH} 8.5$ ) for $1 \mathrm{~h}$ at room temperature. The conjugate was purified by extensive dialysis. To evaluate lysosome function, macrophages were incubated with $2 \mu \mathrm{g} / \mathrm{mL}$ of lysosome function indicator for $1 \mathrm{~h}$, washed with PBS, and suspended using CellStripper (25056CI, Corning). To evaluate lysosomal pH, cells were incubated for $18 \mathrm{~h}$ with $1 \mathrm{mg} / \mathrm{mL}$ FITC-TAMRA dextran (D1951, Thermofisher Scientific) followed by a $4 \mathrm{~h}$ chase period in absence or presence of $10 \mu \mathrm{M}$ Bafilomycin A1 (B1793, Sigma-Aldrich) for the indicated times. In all experiments, 10,000 cells were analyzed by flow cytometry with a LSRII device (BD) using the following lasers and filters: $488 \mathrm{~nm}$ excitation and 515/20 nm emission (FITC and Bodipy), $639 \mathrm{~nm}$ excitation and 660/20 $\mathrm{nm}$ emission (Alexa647) and $532 \mathrm{~nm}$ excitation and 575/26 nm emission (TAMRA). Flowjo v10.6.1 software (www.flowjo.com) was used to export data for each cell for ratiometric analyses.

CRISPR/Cas9 Gpnmb knockout. RAW264.7 macrophages (obtained from ATCC, \# TIB-71) were cultured in DMEM supplemented with 10\% FBS. In order to generate a Gpnmb knockout cell line we designed two sgRNAs using CRISPOR ${ }^{43}$ that spanned the entire Gpnmb gene, such that excision between their target sites would lead to a $21.9 \mathrm{~kb}$ deletion (Table 3). $1 \times 10^{6}$ RAW264.7 cells were co-transfected via nucleofection (Amaxa) with the two mouse Gpnmb sgRNAs (Synthego, $0.6 \mathrm{nM}$ each), which were pre-incubated with 0.07 nM Cas9 protein (Synthego). Transfected cells were plated in 96-well dishes to approximately 1 cell/well then clonally expanded. Clones were screened via Western Blot to find cells without GPNMB protein expression.

Western blot. AKR/J, AKRg-, DBA/2J, DBA $/ 2 \mathrm{~g}^{+}$macrophages, and RAW macrophages with or without CRISPR/Cas9 Gpnmb editing were lysed in RIPA buffer and equal protein levels loaded on 4-20\% tris-glycine gels. After transfer, membranes were probed with antibodies against GPNMB (AF2330, R\&D Systems) and GAPDH (FL-335, Santa Cruz). 
QTL mapping analysis. QTL mapping of macrophage lysosome function ( $\mathrm{Mlfm}$ ) from 120 out of 122 $\mathrm{AKR} / \mathrm{J} \times \mathrm{DBA} / 2 \mathrm{~J}$ F4 BMDMs (the other 2 lines did not yield a sufficient number or viable cells) was performed using R/qtl software, with the final genotype and phenotype data formatted for analysis in the Supplemental Table S1 ( $95^{\text {th }}$ percentile values) and Table S2 (median values) ${ }^{44}$. The "scanone" function was utilized using Haley-Knott regression by specifying the "method" argument as "hk". Genome-wide p-values were ascertained via permutation analysis, using 10,000 permutations by specifying the "n.perm" argument in the "scanone" function. QTL 90\% confidence intervals were calculated using the 1-LOD drop off method. The credible interval for the Mlfm1 locus was also determined by using the Bayesian credible interval ("bayesint") function in R/qtl, with the "prob" argument set at 0.95 . Since Mlfm1 had a significantly higher peak LOD score than any other locus, QTL mapping was performed using the genotype from the best associated $M l f m 1$ marker as an additive covariate ("addcovar") in the "scanone" function of R/qtl. The Mlfml corrected data were subjected to 10,000 permutation analyses to determine genome-wide $\mathrm{p}$-values. To aid in prioritizing candidate genes, a custom $\mathrm{R}$ function termed "flank_LOD" was written (http://www.github.com/BrianRitchey/qtl). This "flank_LOD" function utilizes the "find.flanking" function in R/qtl and returns the LOD score of the nearest flanking marker for a given candidate gene position based on "scanone" output data. Genes in a QTL interval were determined by custom written R functions ("QTL_gene" and "QTL_summary") which utilized publicly available BioMart data from Mouse Genome Build 37. A custom written R function ("pubmed_count") which utilized the rentrez package in R was used to determine the number of PubMed hits for Boolean searches of gene name and terms of interest. Custom written R functions ("sanger_AKRvDBA_missense_genes" and "missense_for_provean") were used to determine the number of non-synonymous mutations between AKR/J and DBA/2J in QTLs, as documented by the Wellcome Trust Sanger Institute's Query SNP webpage for NCBIm37 (https://www.sanger.ac.uk/sanger/Mouse_ SnpViewer/rel-1211). Custom written VBA subroutines ("Provean_IDs" and "Navigate_to_PROVEAN") were used to automate PROVEAN software (http://provean.jcvi.org/seq_submit.php) queries for functional effects of missense mutations in each QTL, with rentrez functions utilized to retrieve dbSNP and protein sequence data. Ultimately, custom R code was used to generate output tables. Deleterious mutations were designated as defined by PROVEAN parameters ${ }^{45}$. Custom code can be found at http://www.github.com/BrianRitchey/qtl.

Statistics. Large data sets were tested for normal distributions and passed, thus parametric statistics were used. Comparison of two conditions was performed by two-tailed student t-test, and comparison of multiple conditions was performed by ANOVA with Tukey or linear trend posttest. All data are shown as mean \pm S.D. Statistics were performed using GraphPad Prism software v9.0.0 (www.graphpad.com).

Ethics approval. All mouse studies were approved by the Cleveland Clinic Institutional Animal Care and Use Committee.

\section{Data availability}

All primary data from this work is available from the corresponding author upon request.

\section{Code availability}

Custom R code can be found at http://www.github.com/BrianRitchey/qtl.

Received: 30 October 2020; Accepted: 30 April 2021

Published online: 13 May 2021

\section{References}

1. Moore, K. J. \& Tabas, I. Macrophages in the pathogenesis of atherosclerosis. Cell 145, 341-355. https://doi.org/10.1016/j.cell.2011. 04.005 (2011).

2. Ouimet, M. et al. Autophagy regulates cholesterol efflux from macrophage foam cells via lysosomal acid lipase. Cell Metab. 13, 655-667. https://doi.org/10.1016/j.cmet.2011.03.023 (2011).

3. Smith, J. D. et al. In silico quantitative trait locus map for atherosclerosis susceptibility in apolipoprotein E-deficient mice. Arterioscler. Thromb. Vasc. Biol. 23, 117-122. https://doi.org/10.1161/01.atv.0000047461.18902.80 (2003).

4. Robinet, P., Ritchey, B. \& Smith, J. D. Physiological difference in autophagic flux in macrophages from 2 mouse strains regulates cholesterol ester metabolism. Arterioscler. Thromb. Vasc. Biol. 33, 903-910. https://doi.org/10.1161/ATVBAHA.112.301041 (2013).

5. Hai, Q. et al. Quantitative trait locus mapping of macrophage cholesterol metabolism and CRISPR/Cas9 editing implicate an ACAT1 truncation as a causal modifier variant. Arterioscler. Thromb. Vasc. Biol. 38, 83-91. https://doi.org/10.1161/ATVBAHA. 117.310173 (2018).

6. Berisha, S. Z., Hsu, J., Robinet, P. \& Smith, J. D. Transcriptome analysis of genes regulated by cholesterol loading in two strains of mouse macrophages associates lysosome pathway and ER stress response with atherosclerosis susceptibility. PLoS ONE 8, e65003. https://doi.org/10.1371/journal.pone.0065003 (2013).

7. Hsu, J. \& Smith, J. D. Genetic-genomic replication to identify candidate mouse atherosclerosis modifier genes. J. Am. Heart Assoc. 2, e005421. https://doi.org/10.1161/JAHA.112.005421 (2013).

8. Anderson, M. G. et al. Mutations in genes encoding melanosomal proteins cause pigmentary glaucoma in DBA/2J mice. Nat. Genet. 30, 81-85. https://doi.org/10.1038/ng794 (2002).

9. $\mathrm{Li}$, B. et al. The melanoma-associated transmembrane glycoprotein Gpnmb controls trafficking of cellular debris for degradation and is essential for tissue repair. FASEB J 24, 4767-4781. https://doi.org/10.1096/fj.10-154757 (2010).

10. van der Lienden, M. J. C., Gaspar, P., Boot, R., Aerts, J. \& van Eijk, M. Glycoprotein non-metastatic protein B: An emerging biomarker for lysosomal dysfunction in macrophages. Int. J. Mol. Sci. https://doi.org/10.3390/ijms20010066 (2018).

11. Kramer, G. et al. Elevation of glycoprotein nonmetastatic melanoma protein B in type 1 Gaucher disease patients and mouse models. FEBS Open Bio 6, 902-913. https://doi.org/10.1002/2211-5463.12078 (2016).

12. Marques, A. R. et al. Gpnmb is a potential marker for the visceral pathology in Niemann-pick type C disease. PLoS ONE 11, e0147208. https://doi.org/10.1371/journal.pone.0147208 (2016). 
13. Ripoll, V. M., Irvine, K. M., Ravasi, T., Sweet, M. J. \& Hume, D. A. Gpnmb is induced in macrophages by IFN-gamma and lipopolysaccharide and acts as a feedback regulator of proinflammatory responses. J. Immunol. 178, 6557-6566. https://doi.org/10.4049/ jimmunol.178.10.6557 (2007).

14. Emanuel, R. et al. Induction of lysosomal biogenesis in atherosclerotic macrophages can rescue lipid-induced lysosomal dysfunction and downstream sequelae. Arterioscler. Thromb. Vasc. Biol. 34, 1942-1952. https://doi.org/10.1161/ATVBAHA.114.303342 (2014).

15. Maquat, L. E. Nonsense-mediated mRNA decay: Splicing, translation and mRNP dynamics. Nat. Rev. Mol. Cell Biol. 5, 89-99. https://doi.org/10.1038/nrm1310 (2004).

16. Howell, G. R. et al. Absence of glaucoma in DBA/2J mice homozygous for wild-type versions of Gpnmb and Tyrp1. BMC Genet. 8, 45. https://doi.org/10.1186/1471-2156-8-45 (2007).

17. Smith, J. D. et al. Atherosclerosis susceptibility loci identified from a strain intercross of apolipoprotein E-deficient mice via a high-density genome scan. Arterioscler. Thromb. Vasc. Biol. 26, 597-603. https://doi.org/10.1161/01.ATV.0000201044.33220.5c (2006).

18. Bhasin, J. M. et al. Sex specific gene regulation and expression QTLs in mouse macrophages from a strain intercross. PLoS ONE 3, e1435. https://doi.org/10.1371/journal.pone.0001435 (2008).

19. Meng, H. et al. Identification of Abcc6 as the major causal gene for dystrophic cardiac calcification in mice through integrative genomics. Proc. Natl. Acad. Sci. USA 104, 4530-4535. https://doi.org/10.1073/pnas.0607620104 (2007).

20. Chang, B. et al. Interacting loci cause severe iris atrophy and glaucoma in DBA/2J mice. Nat. Genet. 21, 405-409. https://doi.org/ 10.1038/7741 (1999)

21. Weterman, M. A. et al. $\mathrm{nmb}$, a novel gene, is expressed in low-metastatic human melanoma cell lines and xenografts. Int. J. Cancer 60, 73-81 (1995).

22. Katayama, A. et al. Beneficial impact of Gpnmb and its significance as a biomarker in nonalcoholic steatohepatitis. Sci. Rep. 5, 16920. https://doi.org/10.1038/srep16920 (2015).

23. Lin, L. Y. et al. Systems genetics approach to biomarker discovery: GPNMB and heart failure in mice and humans. G3 (Bethesda) 8, 3499-3506. https://doi.org/10.1534/g3.118.200655 (2018).

24. Maric, G., Rose, A. A., Annis, M. G. \& Siegel, P. M. Glycoprotein non-metastatic b (GPNMB): A metastatic mediator and emerging therapeutic target in cancer. Onco Targets Ther. 6, 839-852. https://doi.org/10.2147/OTT.S44906 (2013).

25. Moloney, E. B., Moskites, A., Ferrari, E. J., Isacson, O. \& Hallett, P. J. The glycoprotein GPNMB is selectively elevated in the substantia nigra of Parkinson's disease patients and increases after lysosomal stress. Neurobiol. Dis. 120, 1-11. https://doi.org/10. 1016/j.nbd.2018.08.013 (2018).

26. Patel-Chamberlin, M. et al. Hematopoietic growth factor inducible neurokinin-1 (Gpnmb/Osteoactivin) is a biomarker of progressive renal injury across species. Kidney Int. 79, 1138-1148. https://doi.org/10.1038/ki.2011.28 (2011).

27. Singh, M. et al. Functional roles of osteoactivin in normal and disease processes. Crit. Rev. Eukaryot Gene Expr. 20, 341-357 (2010).

28. Jarve, A. et al. Adverse left ventricular remodeling by glycoprotein nonmetastatic melanoma protein B in myocardial infarction. FASEB J. 31, 556-568. https://doi.org/10.1096/f.201600613R (2017).

29. Klein, R. F. et al. Mapping quantitative trait loci that influence femoral cross-sectional area in mice. J. Bone Miner. Res. 17, 1752-1760. https://doi.org/10.1359/jbmr.2002.17.10.1752 (2002).

30. Frara, N. et al. Transgenic expression of osteoactivin/gpnmb enhances bone formation in vivo and osteoprogenitor differentiation ex vivo. J. Cell Physiol. 231, 72-83. https://doi.org/10.1002/jcp.25020 (2016).

31. Sheng, M. H. et al. Targeted overexpression of osteoactivin in cells of osteoclastic lineage promotes osteoclastic resorption and bone loss in mice. PLoS ONE 7, e35280. https://doi.org/10.1371/journal.pone.0035280 (2012).

32. Yukata, K., Nikawa, T., Takahashi, M. \& Yasui, N. Overexpressed osteoactivin reduced osteoclastic callus resorption during distraction osteogenesis in mice. J. Pediatr. Orthop. B https://doi.org/10.1097/BPB.0000000000000789 (2020).

33. Abdelmagid, S. M. et al. Mutation in osteoactivin decreases bone formation in vivo and osteoblast differentiation in vitro. Am. J. Pathol. 184, 697-713. https://doi.org/10.1016/j.ajpath.2013.11.031 (2014).

34. Abdelmagid, S. M. et al. Mutation in osteoactivin promotes Receptor Activator of NFkappaB Ligand (RANKL)-mediated osteoclast differentiation and survival but inhibits osteoclast function. J. Biol. Chem. 290, 20128-20146. https://doi.org/10.1074/jbc.M114. 624270 (2015)

35. DeBoever, C. et al. Large-scale profiling reveals the influence of genetic variation on gene expression in human induced pluripotent stem cells. Cell Stem Cell 20, 533.e7-546.e7. https://doi.org/10.1016/j.stem.2017.03.009 (2017).

36. Warren, C. R. \& Cowan, C. A. Humanity in a dish: Population genetics with iPSCs. Trends Cell Biol. 28, 46-57. https://doi.org/10. 1016/j.tcb.2017.09.006 (2018).

37. Warren, C. R. et al. Induced pluripotent stem cell differentiation enables functional validation of GWAS variants in metabolic disease. Cell Stem Cell 20, 547.e7-557.e7. https://doi.org/10.1016/j.stem.2017.01.010 (2017).

38. Knight, K. R., Vairo, G. \& Hamilton, J. A. Regulation of pinocytosis in murine macrophages by colony-stimulating factors and other agents. J. Leukoc. Biol. 51, 350-359 (1992).

39. Austin, P. E., McCulloch, E. A. \& Till, J. E. Characterization of the factor in L-cell conditioned medium capable of stimulating colony formation by mouse marrow cells in culture. J. Cell Physiol. 77, 121-134. https://doi.org/10.1002/jcp.1040770202 (1971).

40. Fraenkel-Conrat, H. Methods for investigating the essential group for enzyme activity. Methods Enzymol. 4, 247-269 (1957).

41. Basu, S. K., Goldstein, J. L., Anderson, G. W. \& Brown, M. S. Degradation of cationized low density lipoprotein and regulation of cholesterol metabolism in homozygous familial hypercholesterolemia fibroblasts. Proc. Natl. Acad. Sci. USA 73, 3178-3182 (1976).

42. Markwell, M. A., Haas, S. M., Bieber, L. L. \& Tolbert, N. E. A modification of the Lowry procedure to simplify protein determination in membrane and lipoprotein samples. Anal. Biochem. 87, 206-210 (1978).

43. Haeussler, M. et al. Evaluation of off-target and on-target scoring algorithms and integration into the guide RNA selection tool CRISPOR. Genome Biol. 17, 148. https://doi.org/10.1186/s13059-016-1012-2 (2016)

44. Broman, K. W., Wu, H., Sen, S. \& Churchill, G. A. R/qtl: QTL mapping in experimental crosses. Bioinformatics 19, 889-890 (2003).

45. Choi, Y. \& Chan, A. P. PROVEAN web server: A tool to predict the functional effect of amino acid substitutions and indels. Bioinformatics 31, 2745-2747. https://doi.org/10.1093/bioinformatics/btv195 (2015).

\section{Author contributions}

P.R. and J.D.S. conceived and designed the study. P.R., B.R., S.W.L. A.M.A., S.D., E.S. and C.A.T. performed the research and analyzed the data. P.R., S.W.L. and J.D.S. wrote and edited the manuscript. All authors agree the order of author listing and read the final manuscript. All authors approved submission of the final manuscript.

\section{Funding}

This work was funded by the National Institutes of Health grant P01HL029582 to J.D.S and an American Heart Association award 15SDG25310009 to P.R. 


\section{Competing interests}

The authors declare no competing interests.

\section{Additional information}

Supplementary Information The online version contains supplementary material available at https://doi.org/ 10.1038/s41598-021-89800-5.

Correspondence and requests for materials should be addressed to J.D.S.

Reprints and permissions information is available at www.nature.com/reprints.

Publisher's note Springer Nature remains neutral with regard to jurisdictional claims in published maps and institutional affiliations.

(c) (1) Open Access This article is licensed under a Creative Commons Attribution 4.0 International License, which permits use, sharing, adaptation, distribution and reproduction in any medium or format, as long as you give appropriate credit to the original author(s) and the source, provide a link to the Creative Commons licence, and indicate if changes were made. The images or other third party material in this article are included in the article's Creative Commons licence, unless indicated otherwise in a credit line to the material. If material is not included in the article's Creative Commons licence and your intended use is not permitted by statutory regulation or exceeds the permitted use, you will need to obtain permission directly from the copyright holder. To view a copy of this licence, visit http://creativecommons.org/licenses/by/4.0/.

(C) The Author(s) 2021 